\title{
Antimicrobial resistance of Escherichia coli and Staphylococcus aureus isolated from food handler's hands
}

\begin{abstract}
The aim of this study was to determine the antimicrobial resistance of Escherichia coli and Staphylococcus aureus isolates from food handlers' hands at primary schools in Hulu Langat district, Selangor (Malaysia). Disc diffusion methods were used to examine the antimicrobial resistance of the bacteria by using ten types of antibiotic discs with different concentrations. The results show that the prevalence of S. aureus (65.88-74.12\%) was far higher than the prevalence of E. coli (9.41-14.12\%). The percentage isolates of E. coli that were resistant to the antibiotics was $85.71 \%$ Penicillin and Chloramphenicol, $57.14 \%$ SulfamethoxazoleTrimethoprim, Ampicillin and Trimethoprim, 28.57\% Kanamycin and Tetracycline and 14.29\% Ciprofloxacin. All of the isolates had shown susceptible to Gentamicin and Nitrofurantoin. For S. aureus, the percentage isolates that were resistant to the antibiotics was 72.30\% Ampicillin, 53.38\% Penicillin, 4.73\% Nitrofurantoin, $1.35 \%$ Chloramphenicol and Trimethoprim and $0.68 \%$ Kanamycin and Tetracycline. None of the isolates had shown resistant to Ciprofloxacin, Sulfamethoxazole-Trimethoprim and Gentamicin. Multidrug resistant Escherichia coli represented a high percentage $(85.71 \%)$ of the total positive strains revived whereas multidrug resistant $S$. aureus strains were only $5.41 \%$ of the total positive strains. The existence of multidrug resistant bacteria is quite worrying as they may pose serious threat to the patients. Hence, the microbiological quality of food handlers' hands from foodservice operations should be maintained in a good condition to reduce the existence of multidrug resistance bacteria.
\end{abstract}

Keyword: Antibiotic discs; Escherichia coli; Food handlers' hands; Multidrug resistant bacteria; Staphylococcus aureus 